\title{
Customized Automation of Routine EPMA Analyses Using Vendor-Supplied APIs
}

\author{
Daniel Ruscitto $^{1}$, Nathaniel McKeever ${ }^{2}$ and Alberto Santamaria-Pang ${ }^{3}$
}

${ }^{1}$ Materials Organization, GE Research, Niskayuna, New York, United States, ${ }^{2}$ Materials Organization, GE Research, United States, ${ }^{3}$ Artificial Intelligence, GE Research, United States

Inspection of materials for defects and anomalies in high-throughput industrial settings require the collection of dependable, high quality data acquired at a rapid pace. Human errors related to sample tracking and data management along with unintentional deviations from established protocols must be minimized. We have developed a custom system designed to accelerate acquisition of routine electron microprobe analyses by implementing computer vision to track and analyze features of interest. This system's built-in modularity provides a solution that is applicable to various material systems, thereby maintaining flexibility in an industrial research setting where projects and samples are everchanging. Inspections performed using an electron microprobe typically involve one to three mounts in the machine at one time (depending on the size of the sample), each with multiple locations of interest. Numerous features are then analyzed within each location. For a typical mount containing 10 locations of interest, we estimate an average total analysis time (from preparation of the mount to reporting) to be $\sim 400$ minutes. Of that total, $\sim 25 \%$ of the time spent is devoted to point selection by the operator (Figure 1); a task that could easily be automated. Our system is built on a JEOL 8530F electron probe microanalyzer running Probe for EPMA (PFE) software with an attached Thermo Scientific Noran System 7 scan generator. A customized user interface communicates with the system through two APIs (application programming interfaces): Probe Software's Remote Automation Interface [1] and Thermo Scientific's Microanalysis Portal [2]. Two APIs were necessary to overcome two main challenges: (1) slow image acquisition (minimum pixel dwell $\sim 200 \mathrm{msec}$ ) using the JEOL 8520/8530 interface with PFE Remote (version 12.6.2); and (2) lack of beam deflection commands available using Thermo's TEPortal (version 4.0.70). Additionally, a flatbed scanner is attached to the computer running PFE and used to create a reference image for sample navigation. For a typical inspection, the operator first acquires an image of the sample prior to insertion into the microprobe. This scanned image is registered to EPMA stage coordinates and is used to select locations of interest as well as navigation. Once general locations of interest have been set, the program will return to the first location, perform an auto-focus to adjust sample height, and then collect an electron image at high magnification ( >3000X). Customized image recognition software identifies features of interest in the electron image and then uses beam deflection to focus on each feature for measurement. Count rate data and images are stored in a data structure relating all data to optical scanned image and stage coordinate/fiducial information. The program then moves on to the next location specified on the mount. The automated system can achieve a $\sim 30 \%$ decrease in total time per mount and a $\sim 40 \%$ decrease in time that an operator must spend at the instrument (Figure 2). Further time savings are gained for some analyses through modification of the acquisition protocol (e.g., removing background measurements; relying on calibration curves; etc.) Additionally, offline versions of the program can be used by scientists and engineers to specify exact points of interest on a given mount and saved as a file. This file can then be used to guide an operator when they load the mount into the machine and load the previously saved file to investigate regions specified by experts. The current image analysis detection algorithms implement a classifier to: i) segment foreground areas of interest by modeling the brightest and darkest features within a field of view, and ii) exclude porosity and scratches. With the democratization 
of machine learning and deep learning platforms (e.g., scikit-learn, PyTorch), the ability to implement more complex feature detection algorithms is feasible. We will discuss potential future software improvements that leverage machine learning to augment the system's modularity and utility.

TASK BREAKDOWN FOR EPMA (TIME)

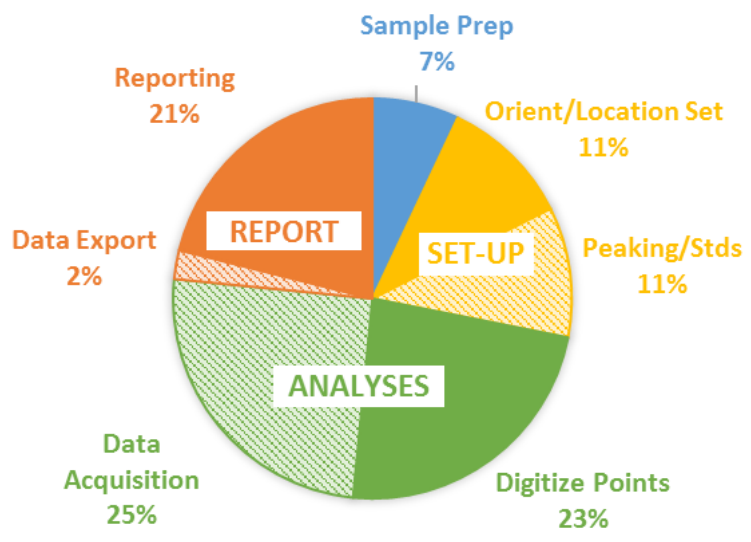

Figure 1. Figure 1. Task breakdown in time (\%) for a typical EPMA mount containing 10 locations of interest and 8 points analyzed per location. 'Sample Prep' includes surface cleaning and coating and does not include sample sectioning or setting of epoxy. Hash-filled sections denote automated tasks using vendor-supplied software. Filled sections show tasks performed by an operator.

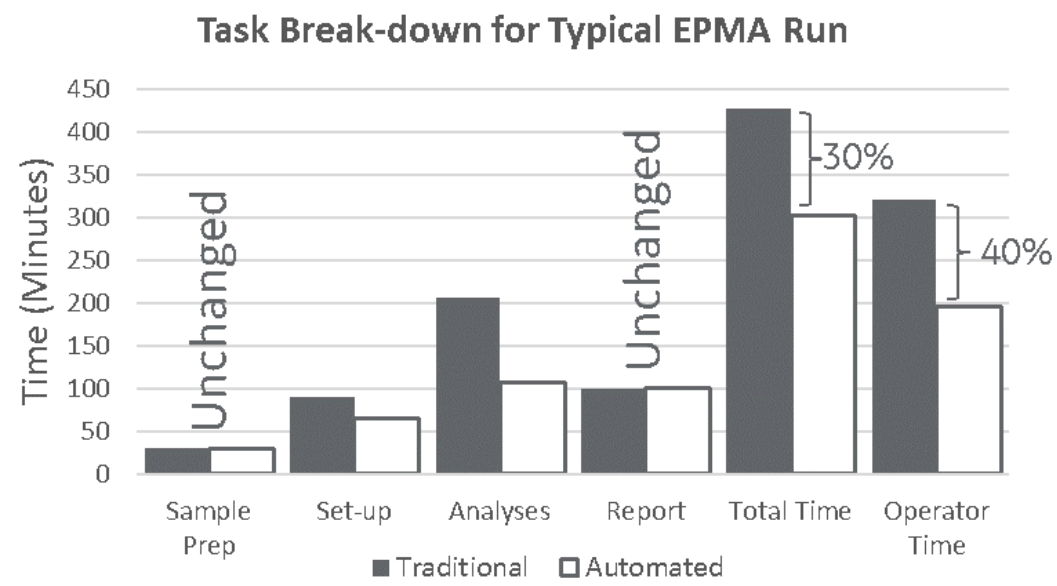

Figure 2. Figure 2. Histograms comparing the time required (in minutes) to complete various tasks between the original or 'Traditional' method and the 'Automated' system for a typical EPMA mount containing 10 locations of interest and 8 points analyzed per location.

References

[1] Donovan, J.J. (2016), Remote Automation COM Server v. 11.7.1 User Guide and Reference. Eugene, OR, USA: Probe Software, Inc.

[2] Thermo Fisher Scientific Inc. (2016), Microanalysis Portal Client Applications User Guide (v.4.0.70). Madison, WI, USA: Thermo Fisher Scientific. 